Available online at GSC Online Press Directory

GSC Biological and Pharmaceutical Sciences

e-ISSN: 2581-3250, CODEN (USA): GBPSC2

Journal homepage: https://www.gsconlinepress.com/journals/gscbps

(RESEARCH ARTICLE)

\title{
Toxic and repellent potentials of different plant oils and new chemistry insecticides against Tribolium castaneum
} \author{
Bushra ${ }^{3}$ \\ ${ }^{1}$ Department of Entomology, University of Agriculture, Faisalabad. \\ 2 Department of Entomology, Bahaudin Zakariya University, Multan. \\ ${ }^{3}$ Department of zoology wild life \& fisheries, University of Agriculture, Faisalabad.
}

Gulzar Muhammad Umair 1, ${ }^{*}$, Rubab Sana 2, Tariq Arbab ${ }^{3}$, Awais Muhammad 1, Saif ul Islam ${ }^{2}$ and Moon

Publication history: Received on 01 April 2020; revised on 18 April 2020; accepted on 07 May 2020

Article DOI: https://doi.org/10.30574/gscbps.2020.11.2.0081

\begin{abstract}
Stored grains commodities are attacked by a variety of beetles and larvae of lepidopteron insect pest of them, Tribolium castaneum (Herbst) is a severe insect pest of stored commodities throughout the world. This insect is widely spreading various climatic regions throughout the world. Results of bioassay with Spinetoram exposed that maximum larval mortality was detected $88.87 \%$ and $44.04 \%$ after " $48 \mathrm{hr}$ " and " $72 \mathrm{hr}$ ". Exposure time period after $0.03 \%$ concentration, correspondingly. Minimum mortality $14.17 \%$ after " $24 \mathrm{hr}$ " at same concentration. At lower concentration $0.02 \%$, maximum mortality $61.96 \%$ after " 72 hr", 31.65\% after " 48 hr" of exposure time period. Minimum mortality of $15.02 \%$ was observed after " $24 \mathrm{hr}$ ". At lowest concentration $0.01 \%$, maximum mortality $60.38 \%$ was recorded at "72 hr", $28.22 \%$ after "48 hr". Whereas, minimum mortality $8.41 \%$ was detected after " $24 \mathrm{hr}$ ". The mortality was increased by increased the concentration of spinetoram and exposure time. Repellent activities of acetone based plant extracts of Eruca sativa, Azadirachta indica and Ricinus communis against the insect pest of stored grain commodities, Tribolium castaneum. Various concentrations $(5,10$ and 15\%) of the plant extracts were applied on the filter papers in the bioassay experiments and after the release of $15 \mathrm{~d}$ old beetles, repellency was evaluated after fixed intervals $(12,24)$. Increased repellency was found at increased concentrations of plants. The concentration interaction and plant extracts findings for the treatments remained momentous. The generally findings exposed raised death rate with raise the doses and time period.
\end{abstract}

Keywords: Eruca sativa; Azadirachta indica; Ricinus communis; T. castaneum

\section{Introduction}

Stored grains commodities are attacked by a variety of beetles and larvae of lepidopteron insect pets. Of them, Tribolium castaneum (Herbst) is a severe insect pest of stored commodities throughout the world. The larvae and adults feed actively on extensive range of long term commodities such as cereals, spices, beans etc. Mainly attack the germ part of seed leading to its poor germination [1]. Huge infestation occurs in favorable conditions (hot and humid conditions). Moreover, T. castaneum attacks in enormous number, leave behind shredded skins, secrete quinines and other toxic substances [2]. This insect is widely spreading various climatic regions throughout the world [3]. Total annual postharvest deficits of stored cereals credited to various natural factors in the storages range between 10-20\% of overall development [4]. Traditional insecticides (fumigants and other synthetic insecticides) have been in practice for the stored grains insect pest management. But excess, repeated and injudicious use of these pesticides poses a lot of problems to humans and natural environment [5]. Use of these fumigants has results in soil contamination, resistance development in the insects (Phosphine resistance) in insects [6]. Indiscriminate use of organophosphates produces

\footnotetext{
* Corresponding author: Gulzar Muhammad Umair
} 
resistance in a number of stored grain insect pests. After that, the use of these organophosphates was decreased [7]. Therefore, there is need to develop and use those pesticides which are environment friendly, less toxic to humans and can control store grain insect pest efficiently. Due to toxic and harsh nature of the fumigants, adverse effects have occurred on human beings and natural enemies such as predator, parasites and parasitoids [8]. All plant derived substances have repellent as well as feeding deterrence properties [8]. The insecticidal action of wide-ranging plant extracts plus more generally of some plant based products chemicals established fact and their utilize was looked after for a large number of previous time throughout all the farming parts of the planet [9]. Plant extracts can be potential and reliable sources for the effective management of stored products insect pests [10]. Many plants have been used in past a now a day against different insect pests' species. Plant materials have repellent effects against stored products insect pests [11]. Plants belong have many toxic substance and volatile compounds which can be explored for the efficient management of insect pests [12]. Scented oils from aromatic plants have been reported to have feeding deterrence [13] and toxic effects can be applied as potential and sustainable alternatives to synthetic insecticides in IPM program for efficient management of stored grains insect pests [14]. Besides these many other researchers have also documented the repellent and toxic effects of different plant materials against insects. The discovery of new chemistry insecticides has opened new horizons in insect pest management. New chemistry insecticides are the microorganisms derived insecticides and have been applied for the management of stored grain insect pests as well as filed insect pests [15]. These insecticides are eco-friendly, have comparatively less toxic effects and have low mammal toxicity compared to other synthetic insecticides [16].

The objectives of study is to investigate the toxic and repellent potentials of three plant oils (Azadirachta indica, Ricinus communis and Eruca sativa) and new chemistry insecticide against Tribolium castaneum.

\section{Material and methods}

\subsection{Collection and mass rearing of the bioassay insects}

Adults of Tribolium castaneum were collected from different grains handlings and storage localities (grain markets) and brought back for mass reared in the laboratory to get homogenous insect culture of the test insect pest. Sterilized plastic jars (having $1.0 \mathrm{~kg}$ capacity) were used for mass rearing insect culture. To confined (avoid the escape) the population of the tested insect, plastic jars were covered with muslin cloth, tightened with rubber bands. The insects were reared on wheat grains flour (as diet). Counted number of adults (100 adults) was released in the plastic jars and place under optimal growth conditions $\left(30+2{ }^{\circ} \mathrm{C}\right.$ and $65+5 \%$ R.H) of the insect. After 3 days, the released beetles were shifted to other jars. The sieved flour containing eggs was again placed into the plastic jars for rearing of the insect till achievement of same age adults, to be used for bioassays.

\subsection{Collection of plants parts for oil extraction}

Seeds of Eruca sativa, Azadirachta indica and Ricinus communis were gathers from different experimental fields in UAF, Faisalabad. The seeds were washing by fresh water and shade dried in the laboratory. Then ground into powders with the help of electrical grinder. Plant oils were extracted against acetone in Soxhlet apparatus in 1:5 ratios of each plant powder (g) and acetone (dipping 50 gram of plant powder in $250 \mathrm{ml}$ acetone). Extracted oils of the selected plants were poured into small cleaned reagent bottles, air tightened and then stored in refrigerator at $4{ }^{\circ} \mathrm{C}$ for further experimentations. New chemistry insecticides were purchased from spray market, located in Faisalabad.

\subsection{Mortality Bioassays}

Different concentrations $(5,10$ and $15 \%$ ) of each of the plant oil and $0.01,0.02$ and $0.03 \%$ new chemistry insecticide were prepared in acetone and applied on filter papers, separately. Thirty adults of T. castaneum (from homogenous population) were bio-assayed. After treatments application, all the experimental units (petri-dish) were placed in cool incubators and data regarding mortality of the test insect pest were recorded after 24,48 , and "72 hr" of the post treatment application.

\subsection{Repellency bioassay}

Surface area preference method was followed in evaluating the repellent potential of the plant extracts and new chemistry insecticides. Half of each filter paper was treated whilst leaving untreated the second half of each filter paper by different concentrations (as mentioned earlier). Both half of each filter paper was clipped at central point and placed in petri-dishes and data regarding repellency were recorded after 12 and " $24 \mathrm{hr}$ ". 


\subsection{Statistical analysis}

Outcomes of the bioassays (especially mortality data) were corrected by Abbott's formula and then subjected to static software 8.1 for analysis. Comparison of treatments means was done by Tukey's-HSD test (at $\alpha$ 5\%).

\section{Results and discussion}

\subsection{Mortality findings after " $24 \mathrm{hr}$ "}

Table 1 Comparison per cent mean mortality values of Tribolium castaneum after application of altered concentrations of plant oils

\begin{tabular}{ll}
\hline Concentrations (\%) & Per cent Mean mortality \pm SE \\
\hline 5 & $5.13 \pm 1.30 \mathrm{c}$ \\
10 & $2.21 \mathrm{~b}$ \\
15 & $15.90 \mathrm{a}$ \\
\hline
\end{tabular}

Data in Table (1) displayed lowest mean per cent mortality $5.13 \%$ at 5\% concentration and $15.90 \%$ mortality was recorded at $15 \%$ concentration of the plant oils, used. From findings of the bioassay we can conclude that per cent mean per cent mortality of $T$. castaneum was found significantly affected by plant oil concentrations.

Table 2 Comparative mean percentage mortality values of Tribolium castaneum at different concentrations

\begin{tabular}{ll}
\hline Plant oils x Concentrations (\%) & (\%) Mean Mortality \pm SE \\
\hline Ricinus communis x 5 & $3.15 \pm 1.06 \mathrm{e}$ \\
Ricinus communis x 10 & $7.36 \pm 1.92 \mathrm{~d}$ \\
Ricinus communis x 15 & $13.52 \pm 1.64 \mathrm{~cd}$ \\
Eruca sativa x 5 & $8.16 \pm 2.19 \mathrm{~d}$ \\
Eruca sativa x 10 & $15.67 \pm 1.96 \mathrm{~cd}$ \\
Eruca sativa x 15 & $22.42 \pm 1.25 \mathrm{~b}$ \\
Azadirachta indica x 5 & $9.62 \pm 2.57 \mathrm{~d}$ \\
Azadirachta indica x 10 & $18.71 \pm 2.03 \mathrm{c}$ \\
Azadirachta indica x 15 & $38.16 \pm 2.03 \mathrm{a}$ \\
\hline
\end{tabular}

Table 2 displayed that relatively highest mortality 38.16\% was recorded at $15 \%$ concentration of Azadirachta indica with exposure period of " $24 \mathrm{hr}$ ". Followed $22.42 \%$ with extract of Eruca sativa and $13.52 \%$ by extract of Ricinus communis at same concentrations. At 10\% concentration, mean percentage mortality values were $18.71,15.67$ and $7.36 \%$ respectively. Comparatively low mortality values $9.62,8.16$ and $3.15 \%$ were examined at $5 \%$ concentrations of A. indica, E. sativa and $R$. communis correspondingly. From the outcomes we can determined that there was a slowly increase in mortality values with rise in plant extract concentrations, used.

\subsection{Mortality data after exposure of " $48 \mathrm{hr}$ "}

Table 3 Comparison of the mean percentage mortality of Tribolium castaneum after exposure to different concentrations of plant oils

\begin{tabular}{ll}
\hline Concentrations (\%) & Mean percentage mortality \pm SE \\
\hline 5 & $8.34 \pm 2.04$ \\
10 & $20.16 \pm 1.86$ \\
15 & $32.13 \pm 3.07$ \\
\hline
\end{tabular}


Tabulated data (3) represents the lethal effects of different concentrations of three different oil against Tribolium castaneum. Highest mortality (32.13\%) at 15\% was noticed. The mortality was 8.34 and $20.16 \%$ at 5 and $10 \%$ concentration, respectively. From the outcomes it is concluded that mortality only increased with increase in concentrations of the three plant oils and also displayed that concentration has notice-worthy effect on \% mean per cent mortality of T. castaneum.

Table 4 Interaction effect (Plant oils x Concentrations) for mean percentage mortality of Tribolium castaneum

\begin{tabular}{ll}
\hline Plant oils $\mathrm{x}$ Concentrations (\%) & (\%) Mean Mortality \pm SE \\
\hline Ricinus communis $\times 5$ & $6.34 \pm 1.52 \mathrm{~g}$ \\
Ricinus communis $\times 10$ & $13.15 \pm 1.52 \mathrm{def}$ \\
Ricinus communis $\times 15$ & $18.17 \pm 1.52 \mathrm{efg}$ \\
Eruca sativa $\times$ 5 & $9.67 \pm 0.34 \mathrm{fg}$ \\
Eruca sativa $\times 10$ & $21.34 \pm 1.34 \mathrm{de}$ \\
Eruca sativa $\times 15$ & $29.01 \pm 1.27 \mathrm{bc}$ \\
Azadirachta indica $\times$ 5 & $12.12 \pm 1.54 \mathrm{~cd}$ \\
Azadirachta indica $\times 10$ & $31.98 \pm 1.87 \mathrm{~b}$ \\
Azadirachta indica $\times 15$ & $49.11 \pm 2.42 \mathrm{a}$ \\
\hline
\end{tabular}

Table 4 showed the interaction between different concentrations $[5,10$ and 15\%) and different exposure time period. Mean comparison of percentage mortality values of T. castaneum at different concentrations of selected plant extract were highest at maximum concentration. Findings of the mortality bioassay indicated that maximum mortality (49.11\%) at $15 \%$ was recorded by Azadirachta indica. The mean mortality was $31.98 \%$ at $10 \%$ concentration and $12.12 \%$ mortality was observed at 5\% concentration of the plant oil. Oil of Eruca sativa gave mean per cent mortality (27.01\%) at $15 \%$ was recorded. The mean mortality was $21.34 \%$ at $10 \%$ concentration and $9.67 \%$ mortality was observed at $5 \%$ concentration of the E. sativa. Plant oil of Ricinus communis gave relatively mean mortality (18.17\%) at $15 \%$ was recorded whilst least mortality $6.34 \%$ was observed at $5 \%$ concentration of the plant extracts. The given outcome showed that interaction of exposure time and concentration was significant. From results we concluded that there was a gradually increase in mortality values with increase in concentration of plant oils.

\subsection{Mortality data after exposure of " $72 \mathrm{hr}$ "}

Table 5 Comparison of the mean percentage mortality of Tribolium castaneum after exposure to different plant oil concentrations

\begin{tabular}{ll}
\hline Concentrations (\%) & Mean \% mortality \pm SE \\
\hline 5 & $21.61 \pm 2.36 \mathrm{c}$ \\
10 & $30.97 \pm 3.24 \mathrm{~b}$ \\
15 & $44.86 \pm 2.56 \mathrm{a}$ \\
\hline
\end{tabular}

Data Table 5 showed that mortality $21.71 \%$ mean percentage mortality was recorded at $5 \%$ concentration and $44.86 \%$ mortality was observed at $15 \%$ concentration of the plant oils. From results we can conclude that concentration has significant effect on per cent mean per cent mortality of T. castaneum. 
Table 6 Comparative mean percentage mortality of T. castaneum after exposure to various concentrations of plant oils after

\begin{tabular}{ll}
\hline Plant oils x Concentrations (\%) & (\%) Mean Mortality \pm SE \\
\hline Ricinus communis x 5 & $15.32 \pm 2.88$ \\
Ricinus communis x 10 & $25.82 \pm 1.66$ \\
Ricinus communis x 15 & $28.67 \pm 2.64$ \\
Eruca sativa x 5 & $22.46 \pm 2.88$ \\
Eruca sativa x 10 & $42.56 \pm 2.92$ \\
Eruca sativa x 15 & $49.38 \pm 2.89$ \\
Azadirachta indica $\times 5$ & $30.87 \pm 1.66 \mathrm{bc}$ \\
Azadirachta indica x 10 & $35.02 \pm 3.38 \mathrm{ab}$ \\
Azadirachta indica $\times 15$ & $57.81 \pm 3.40 \mathrm{ab}$ \\
\hline
\end{tabular}

Table 6. Exposed that, highest mortality 57.81\% was recorded with $15 \%$ concentration of Azadirachta indica after exposure of "48 hr" followed $49.38 \%$ with extract of Eruca sativa, whilst Ricinus communis gave $28.67 \%$ at $15 \%$ concentrations. Mean percentage mortality of $30.87 \%$ was recorded at $5 \%$ concentration of Azadirachta indica, followed by Eruca sativa $22.46 \%$. Mean mortality of $17.40 \%$ was given by Ricinus communis at $5 \%$ concentration. The lowest mean mortality was found 25.82 at $10 \%$ concentration of R. communis. In case of Azadirachta indica and Eruca sativa 35.02 and $42.56 \%$ mean percentage mortality were observed at $10 \%$ concentration of plant extract. From results we concluded that, there was a gradually increase in mortality values with increase in concentration of plant extracts

\subsection{Mortality data with Spinetoram}

Table 7 Comparison of the mean percentage mortality of Tribolium castaneum after exposure to different concentrations of Spinetoram

\begin{tabular}{ll}
\hline Concentrations (\%) & Larval Emergence \pm SE \\
\hline 0.01 & $32.08 \pm 3.36 \mathrm{~b}$ \\
0.02 & $36.54 \pm 2.98 \mathrm{~b}$ \\
0.03 & $3.24 \mathrm{a}$ \\
\hline
\end{tabular}

Data Table 7 showed that mortality $32.08 \%$ mean percentage mortality was observed at $0.01 \%$ concentration and $49.96 \%$ mortality was observed at $0.03 \%$ concentration of the plant extracts. From results we can conclude that concentration has significant effect on per cent mean per cent mortality of T. castaneum.

Table 8 Comparative mean percentage mortality of Tribolium castaneum after exposure to different concentrations of Spinetoram

\begin{tabular}{ll}
\hline Time x Concentrations (\%) & (\%) Mean Mortality \pm SE \\
\hline $24 \times 0.01$ & $8.42 \pm 2.88 \mathrm{f}$ \\
$24 \times 0.02$ & $15.02 \pm 1.66 \mathrm{de}$ \\
$24 \times 0.03$ & $15.27 \pm 2.64 \mathrm{de}$ \\
$48 \times 0.01$ & $28.32 \pm 3.13 \mathrm{~cd}$ \\
$48 \times 0.02$ & $31.65 \pm 1.65 \mathrm{~cd}$ \\
$48 \times 0.03$ & $45.02 \pm 3.32 \mathrm{bc}$ \\
$72 \times 0.01$ & $59.48 \pm 2.88 \mathrm{ab}$ \\
$72 \times 0.02$ & $62.96 \pm 2.92 \mathrm{~b}$ \\
$72 \times 0.03$ & $89.88 \pm 2.89 \mathrm{a}$ \\
\hline
\end{tabular}

Table 8 showed that maximum mortality $89.88 \%$ was observed with $0.03 \%$ concentration of spinetoram after exposure of "72 hr". Mortality was $62.96 \%$ followed by $31.65 \%$ and 15.02 was observed with $0.02 \%$ concentration of the 
spinetoram. Mortality was 59.48 was observed after "72 hr" at $0.01 \%$ concentration, followed by $28.32 \%$ after "48 hr" and $15.27 \%$ after " $24 \mathrm{hr}$ " of the exposure period of the spinetoram. The $0.02 \%$ and $0.03 \%$ concentrations of the spinetoram showed almost equal mortality results. After "48 hr", mortality was 45.02 at $0.03 \%$ concentrations of spinetoram. From results we concluded that almost all concentration and exposure time intervals were significant there was a gradually increase in mortality values with increase in concentration of spinetoram.

\subsection{Combined mortality data of plant extracts and spinetoram}

Table 9 Combined effects of plant oils and Spinetoram for mean percentage mortality of Tribolium castaneum

\begin{tabular}{ll}
\hline Combined effects & \% Mortality \pm SE \\
\hline Ricinus communis (15\%)+Spinetoram(0.03\%) & $34.28 \pm 2.36 \mathrm{~b}$ \\
Eruca sativa (15\%)+Spinetoram(0.03\%) & $36.53 \pm 2.38 \mathrm{~b}$ \\
Azadirachta indica (15\%)+Spinetoram $(0.03 \%)$ & $3.05 \mathrm{a}$ \\
\hline
\end{tabular}

Data Table 9 showed that mortality 34.28\% mean percentage mortality was observed with Ricinus communis (15\%)+Spinetoram (0.03\%). Mortality of $36.53 \%$ was observed for Eruca sativa (15\%)+Spinetoram (0.03\%). Mortality of $52.47 \%$ was observed by Azadirachta indica (15\%)+Spinetoram (0.03\%). From results we can conclude that combine action of Azadirachta indica + Spinetoram has significant effect on per cent mean per cent mortality of T. castaneum.

Table 10 Comparison of the mean percentage mortality of Tribolium castaneum by combine action of different plant oils at different time periods

\begin{tabular}{ll}
\hline Plant x Time & (\%) Mean Mortality \pm SE \\
\hline Ricinus communis $(15 \%)+$ Spinetoram $(0.03 \%) \times 24$ & $15.24 \pm 2.88 \mathrm{~d}$ \\
Ricinus communis $(15 \%)+$ Spinetoram $(0.03 \%) \times 48$ & $25.17 \pm 2.66 \mathrm{~cd}$ \\
Ricinus communis $(15 \%)+$ Spinetoram $(0.03 \%)$ x 72 & $39.38 \pm 1.71 \mathrm{bcd}$ \\
Eruca sativa $(15 \%)+$ Spinetoram $(0.03 \%) \times 24$ & $16.65 \pm 3.40 \mathrm{~d}$ \\
Eruca sativa $(15 \%)+$ Spinetoram $(0.03 \%) \times 48$ & $33.35 \pm 3.26 \mathrm{bcd}$ \\
Eruca sativa $(15 \%)+$ Spinetoram $(0.03 \%) \times 72$ & $52.86 \pm 3.37 \mathrm{~b}$ \\
Azadirachta indica $(15 \%)+$ Spinetoram $(0.03 \%) \times 24$ & $24.23 \pm 2.91 \mathrm{~cd}$ \\
Azadirachta indica $(15 \%)+$ Spinetoram $(0.03 \%) \times 48$ & $48.33 \pm 2.18 \mathrm{bc}$ \\
Azadirachta indica $(15 \%)+$ Spinetoram $(0.03 \%) \times 72$ & $78.83 \pm 2.91 \mathrm{a}$ \\
\hline
\end{tabular}

Table 10 showed that maximum mortality 78.83\% was observed after exposure of "72 hr". by Azadirachta indica (15\%)+Spinetoram (0.03\%) followed by $52.86 \%$ by Eruca sativa (15\%)+Spinetoram (0.03\%) and 39.38 was observed by Ricinus communis (15\%)+Spinetoram (0.03\%), respectively. Mortality was 48.33 was observed after "48 hr". exposure period, followed by $33.35 \%$ and $25.17 \%$, respectively. While least percentage mean mortality $15.24 \%$ was observed by application of Ricinus communis (15\%)+Spinetoram (0.03\%) after "24 hr" of the exposure period. From results we concluded that combined action of A. indica after "72 hr"s. was most effective than other exposure periods and there was almost a significant increase in mortality values with increase in exposure period.

\subsection{Repellency data after "12 hrs"}

The outcome of the repellency bioassays in Table 11 revealed that repellency was found increased with increase in concentration for all extract of plants. Maximum repellency $71.24 \%$ was recorded at $15 \%$ concentration while minimum $40.60 \%$ was recorded at $5 \%$ concentration. 
Table 11 Comparison of the mean percentage repellency of T. castaneum after exposure to various concentrations of plant oils

\begin{tabular}{ll}
\hline Concentration (\%) & \% Repellency \pm SE \\
\hline 5 & $40.60 \pm 2.98 \mathrm{c}$ \\
10 & $63.45 \pm 2.54 \mathrm{~b}$ \\
15 & $3.11 \mathrm{a}$ \\
\hline
\end{tabular}

Table 12 Comparative Plants x Concentrations for mean percentage repellency against Tribolium castaneum

\begin{tabular}{|c|c|}
\hline Plants x Concentrations (\%) & $\%$ Repellency \pm SE \\
\hline Eruca sativa $\times 5$ & $28.34 \pm 1.13$ \\
\hline Eruca sativa $\times 10$ & $46.23 \pm 2.10$ \\
\hline Eruca sativa $\times 15$ & $53.56 \pm 2.91$ \\
\hline Ricinus communis $\mathrm{x} 5$ & $35.42 \pm 2.61$ \\
\hline Ricinus communis $\mathrm{x} 10$ & $57.13 \pm 2.76$ \\
\hline Ricinus communis $\mathrm{x} 15$ & $65.89 \pm 3.05$ \\
\hline Azadirachta indica x 5 & $46.56 \pm 2.60$ \\
\hline Azadirachta indica $\times 10$ & $60.31 \pm 1.89$ \\
\hline Azadirachta indica $\times 15$ & $69.16 \pm 3.03 a$ \\
\hline
\end{tabular}

Data in Table 12 showed that all extract of plants were significantly different from each other. Repellency was increased with rise in concentration for all extracts of plant. Extracts of Azadirachta indica and Ricinus communis gave highest repellency results with values 69.16 and $65.89 \%$ followed while Eruca sativa gave $53.56 \%$ at $15 \%$ concentration. At $10 \%$ concentrations of the plant extracts gave relatively lower values but comparatively greater values of mean per cent repellency at $5 \%$ concentrations of the test plant extracts.

\subsection{Repellency data after " $24 \mathrm{hr}$ ”}

The outcomes of the repellency bioassays in Table 13 revealed that repellency was found increased with rise in concentration for all plant extracts. Maximum repellency (76.36\%) was observed at $5 \%$ concentrations while minimum $40.60 \%$ was recorded in untreated unit (control treatment).

Table 13 Comparative mean percentage repellency of Tribolium castaneum after exposure to different concentrations of plant oils

\begin{tabular}{ll}
\hline Concentrations (\%) & \% Repellency \pm SE \\
\hline 5 & $40.60 \pm 2.98 \mathrm{c}$ \\
10 & $71.21 \pm 2.49 \mathrm{~b}$ \\
15 & $76.36 \pm 2.33 \mathrm{a}$ \\
\hline
\end{tabular}

Data in Table 14 showed that all plant extracts were significantly different from each other. Repellency was increased with rise in concentration for all plant extracts. Extracts of lemongrass and basil gave similar and maximum repellency results with values $90.00 \%$ followed by Datura $86.56 \%$ and garlic $85.67 \%$ at $15 \%$ concentration. Extracts of marigold gave $80.00 \%$ at similar concentration. Repellency value of $73.32 \%$ was recorded in case of monocarps at $15 \%$ concentration while minimum repellency was recorded in control treatment. Repellency of $43.32 \%$ was recorded in Petunia after " $24 \mathrm{hr}$ ". exposure period. Extracts of mint, basil and petunia, and gave relatively low repellency with values $26.65 \%, 21.66 \%$ and $20.03 \%$, respectively. 
Table 14 Comparative mean percentage repellency of Tribolium castaneum after exposure to different concentrations of plant oils

\begin{tabular}{|c|c|}
\hline Plants x Concentrations (\%) & $\%$ Repellency \pm SE \\
\hline Eruca sativa $\times 5$ & $50.01 \pm 2.77$ \\
\hline Eruca sativa $\times 10$ & $76.67 \pm 3.33$ \\
\hline Eruca sativa $\times 15$ & $86.56 \pm 3.33$ \\
\hline Ricinus communis $\mathrm{x} 5$ & $40.11 \pm 2.67$ \\
\hline Ricinus communis $\mathrm{x} 10$ & $63.01 \pm 2.77$ \\
\hline Ricinus communis $\mathrm{x} 15$ & $70.00 \pm 2.81$ \\
\hline Azadirachta indica x 5 & $56.00 \pm 2.66$ \\
\hline Azadirachta indica $\times 10$ & $83.31 \pm 3.33 \mathrm{ab}$ \\
\hline Azadirachta indica x 15 & $85.67 \pm 2.88 \mathrm{a}$ \\
\hline
\end{tabular}

\section{Discussion}

Results of bioassay with spinetoram exposed that maximum larval mortality was detected $88.87 \%$ and $44.04 \%$ after "48 hr". and "72 hr". exposure time period after $0.03 \%$ concentration, correspondingly. Minimum mortality $14.17 \%$ after " $24 \mathrm{hr}$ ". at same concentration. At lower concentration $0.02 \%$, maximum mortality $61.96 \%$ after " $72 \mathrm{hr}$ "., $31.65 \%$ after "48 hr". of exposure time period. Minimum mortality of $15.02 \%$ was observed after " $24 \mathrm{hr}$ ". At lowest concentration $0.01 \%$, maximum mortality $60.38 \%$ was recorded at " $72 \mathrm{hr}$ "., $28.22 \%$ after "48 hr". whereas, minimum mortality $8.41 \%$ was detected after " $24 \mathrm{hr}$ ". The mortality was increased by increased the concentration of spinetoram and exposure time. Furthermore, interactions of different concentrations and exposure time periods were found highly significant. [1] evaluate the 3 important oils like a E. cardamomum, C. aromaticum and S. aromaticum Merr and Petry alongside the T. castaneum at its different life stages. The findings exhibited that, $C$. aromaticum and E. cardamomum provide more effective for the young once and adults whereas, $S$. aromaticum has lower impact alongside the $T$. castaneum young once and adults. In make contact with fumigation lethal impact on mature and other developmental stages of red flour beetle was additional resilient to clove compared to cinnamon and cardamom oil. After described the efficacy of Azadirachta indica, the [17] assessed the impact of Teminalia chebula, Azadirachta indica, Murraya exotica, Eucalyptus comeldulensis and Trachspermum ammi were evaluated at the rate of 5,10 and $15 \%$ concentrations against the $T$. castaneum. The death rate of tested insects was increased by increased concentration. The mortality trend was found similar to our study.

\subsection{Repellent effects of different plant material for alongside the (T. castaneum) red flour beetle at different exposure time and at different concentration levels}

Our results of repellency against $T$. castaneum are in accordance with [18] who evaluated the laboratory experimentations were conducted for the evaluation of contact and repellent activities of acetone based plant extracts of Eruca sativa, Azadirachta indica and Ricinus communis against the insect pest of stored grain commodities, Tribolium castaneum. Various concentrations (5, 10 and 15\%) of the plant extracts were applied on the filter papers in the bioassay experiments and after the release of $15 \mathrm{~d}$ old beetles, mortality was evaluated after fixed intervals $(12,24)$. Increased mortality was found at increased concentrations of plants. The concentration interaction and plant extracts findings for the treatments remained momentous. The generally findings exposed raised death rate with raise the doses and time period.

\section{Conclusion}

The mortality was increased by increased the concentration of spinetoram and exposure time. Repellent activities of acetone based plant extracts of Eruca sativa, Azadirachta indica and Ricinus communis against the insect pest of stored grain commodities, Tribolium castaneum. Various concentrations $(5,10$ and 15\%) of the plant extracts were applied on the filter papers in the bioassay experiments and after the release of $15 \mathrm{~d}$ old beetles, repellency was evaluated after fixed intervals $(12,24)$. Increased repellency was found at increased concentrations of plants. The concentration 
interaction and plant extracts findings for the treatments remained momentous. The generally findings exposed raised death rate with raise the doses and time period.

\section{Compliance with ethical standards}

\section{Acknowledgments}

Thanks to all the research fellows.

\section{Disclosure of conflict of interest}

No observed conflict of interest among the authors.

\section{References}

[1] Mondal KAMSH. (1994). Flour beetles Tribolium spp. (Coleoptera: Tenebrionidae) aspects and their control. Agri, Zool. Rev, 6, 95-195.

[2] Ogando JO, M Kostyukovsky, U Ravid, JC Matasyoh, AL Deng, EO Omolo, ST Kariuki and E Shaaya. (2008). Bioactivity of Ocimum gratissimum L. oil and two of its constituents against five insect pests attacking stored food products. J. Stored Prod. Res, 44(4), 328-334.

[3] Shafique M, M Ahmad and MA Chaudhry. (2006).Feeding Preference and Development of Tribolium castaneum (Herbst.) in Wheat Products. Pak. J. Zool, 38(1), 27-31.

[4] Phillips TW and JE Throne. (2010). Bio rational approaches to managing stored-product insects. Annual Review Entomol, 55, 375-397.

[5] Koul O, S Walia and GS Dhaliwal. (2008). Essential oils as green pesticides: potential and constraints. Bio pesticides Int, 4, 63-84.

[6] Benhalima H, MQ Chaudhry, KA Mills and NR Price. (2004). Phosphine resistance in stored-product insects collected from various grain storage facilities in Morocco. J. Stored Prod Res, 40(3), 241-249.

[7] Fang L, B Subramanyam and FH Arthur. (2002). Effective of Spinosad on four classes of wheat against five stored products insect. J. Econ. Entomol, 95, 640-650.

[8] Isman MB. (2006). Botanical insecticides, deterrents, and repellents in modern agriculture and increasingly regulated world. Ann. Rev. Entomol, 51, 45-66.

[9] Regnault RC, C Vincent and JT Arnason. (2012). Essential oils in insect control. low-risk products in a high-stakes world. Annual Rev. Entomol, 57, 405-424.

[10] Padin SB, C Fuse, MI Urrutia and GMD Bello. (2013). Toxicity and repellency of nine medicinal plants against Tribolium castaneum in stored wheat. Bull. Insectol, 66(1), 45-49.

[11] Rehman H, M Hasan, Q Ali, M Yasir, S Saleem, S Mirza, HU Shakir, AM Alvi and HM Ahmed. (2017). Potential of three indigenous plants extracts for the control of Tribolium castaneum (Herbst) and Rhyzopertha dominica (Fab.). Pak. Entomol, 40(1), 31-37.

[12] Suthisut D, PG Fields and A Chandrapatya. (2011). Fumigant toxicity of essential oils from three Thai plants (Zingiberaceae) and their major compounds against Sitophilus zeamais and Tribolium castaneum and two parasitoids. J. Stored Prod. Res, 47(3), 222-230.

[13] Taghizadeh R and NM Khani. (2016).Feeding deterrency of two medicinal plant extracts on Tribolium castaneum (Coleoptera: Tenebrionidae). J. Crop Prot, 5(4), 529-539.

[14] Regnault RC, C Vincent and JT Arnason. (2012). Essential oils in insect control. low-risk products in a high-stakes world. Annual Rev. Entomol, 57, 405-424.

[15] Hertlein MB, GD Thompson, B Subramanyam, CG Athanassiou. (2011). Spinosad: a new natural product for stored grain protection. J. Stored Prod. Res, 47, 131-146.

[16] Yee WL and DG Alston. (2012). Behavioral responses, rate of mortality and oviposition of western cherry fruit fly exposed to Malathion, zeta-cypermethrin and Spinetoram. J. Pest Sci., 85(1), 141-151. 
[17] Sagheer M, MU Hasan, M Najam-ul-Hassan, M Farhan, FZA Khan and A Rahman. (2014). Repellent effects of selected medicinal plant extracts against rust-red flour beetle, Tribolium castaneum (Herbst) (Coleoptera: Tenebrionidae). J. Entomol. Zool. Stud, 2(3), 107-110.

[18] Khan HA, Shad SA and Akram W. (2013). Resistance to new chemical insecticides in the house fly, Musca domestica L., from dairies in Punjab, Pakistan. Parasitology research, 112(5), 2049-2054.

\section{How to cite this article}

Gulzar MU, Rubab S, Tariq A, Awais M, Saif uI and Moon B. (2020). Toxic and repellent potentials of different plant oils and new chemistry insecticides against Tribolium castaneum. GSC Biological and Pharmaceutical Sciences, 11(2), 61-70. 